\title{
Aprovechamiento de residuos agroindustriales como alternativa en el mejoramiento de la calidad del ambiente
}

\author{
Use of agro-industrial waste as an alternative to improve the quality of the environment
}

Aproveitamento de resíduos agroindustriais como alternativa para a melhoria da qualidade do meio ambiente

\author{
Santiago Aguiar ${ }^{1}$ \\ saguiar@uea.edu.ec \\ https://orcid.org/0000-0002-1971-7330
}

\author{
Hernán Uvidia ${ }^{1}$ \\ huvidia@uea.edu.ec \\ https://orcid.org/0000-0002-2961-6963
}

\author{
Luis Arboleda ${ }^{2}$ \\ larboleda@espoch.edu.ec \\ https://orcid.org/0000-0001-5541-6239 \\ ${ }^{1}$ Universidad Estatal Amazónica. Pastaza-Ecuador \\ ${ }^{2}$ Escuela Superior Politécnica de Chimborazo, Riobamba, Chimborazo-Ecuador
}

Artículo recibido 28 de octubre 2021 / Arbitrado y aceptado 29 de noviembre 2021 / Publicado 30 de diciembre 2021

\section{RESUMEN}

El aumento del volumen de desechos sólidos agroindustriales, agrícolas y domésticos conlleva una serie de implicaciones relacionadas con aspectos de salubridad y medioambientales. El objetivo de esta investigación es plantear posibles alternativas de aprovechamiento de residuos generados en la agroindustria, a partir del procesamiento de snacks. Estas alternativas de aprovechamiento se formulan a partir de la caracterización fisicoquímica de los residuos, la cual se llevó a cabo por medio de análisis proximal, análisis elemental como materia seca (MS), materia orgánica $(\mathrm{MO})$, proteína bruta $(\mathrm{PB})$, fibra bruta (FB), cenizas, extracto etéreo (EE), extractos libres de nitrógeno (ELN) y energía bruta (EB), se utilizó estadística descriptiva y se determinó la media y desviación estándar. Se encontró que la gran mayoría de los residuos pueden ser aprovechados como materia prima en procesos de producción de alimentación animal. La cáscara de plátano y yuca presentaron el mayor tenor de cenizas $(8,82$ y $8,49 \%)$ respectivamente. Los subproductos agroindustriales de maní sin cáscara, maní con cáscara, cáscara de plátano, cáscara de yuca, cáscara de habas y habas sin cáscara presentaron buen contenido de MS, MO, PB, ELN, EE, Cenizas y EB.

Palabras clave: Residuos agroindustriales; Bioenergéticas; Desarrollo sostenible; Análisis proximal

\section{ABSTRACT}

The increpase in the volumen of agroindustrial, agricultural and domestica salid wastes caries a series of implicaciones related to Health and Environmentally aspects. The objectivo of thais research is to propuse Possible alternatives cor the use of wastes generated in agribusiness, from the processing of snacks. These use alternatives are formulated from the physicochemical characterization of the wastes, which was carried out through proximal analysis, elemental analysis such as dry matter (DM), organic matter (OM), crude protein (CP), fiber crude $(\mathrm{FB})$, ash, ethereal extract (EE), nitrogenfree extracts (ELN) and crude energy (EB), descriptive statistics were used and the mean and standard deviation were determined. It was found that the vast majority of wastes can be used as raw material in animal feed production processes. Banana peel and cassava had the highest ash content (8.82 and $8.49 \%)$ respectively. The agro-industrial by-products of shelled peanuts, shelled peanuts, banana peel, cassava peel, broad bean peel and shelled broad beans presented good content of DM, OM, PB, ELN, EE, Ash and EB.

Key words: Agro-industrial wastes; Bioenergetics; Sustainable development; Proximal analysis

\section{RESUMO}

O aumento do volumen de resíduos sólidos agroindustriais, agrícolas e domésticos acarreta uma série de implicações relacionadas aos aspectos de saúde e meio ambiente. O objetivo desta esquisa é propor possíveis alternativas para o aproveitamento dos resíduos gerados na agroindústria, a partir do processamento de salgadinhos. Essas alternativas de uso são formuladas a partir da caracterização físico-química do resíduo, que foi realizada por meio de análises proximais, análises elementares como matéria seca (MS), matéria orgânica (MO), proteína bruta (CP), fibra bruta $(F B)$, cinzas, extrato etéreo (EE), extratos isentos de nitrogênio (ELN) e energia bruta (EB), foram utilizadas estatísticas descritivas e determinados a média e o desvio padrão. Verificou-se que a grande maioria dos resíduos pode ser utilizada como matéria-prima em processos de produção de ração animal. A casca de banana e a mandioca apresentaram os maiores teores de cinzas (8,82 e $8,49 \%$ ), respectivamente. Os subprodutos agroindustriais de amendoim com casca, amendoim com casca, casca de banana, casca de mandioca, casca de fava e fava com casca apresentaram bons teores de MS, MO, PB, ELN, EE, Cinza e EB

Palavras-chave: Resíduos agroindustriais; Bioenergética; Desenvolvimento sustentável; Análise proximal 
INTRODUCCIÓN

El gran crecimiento de la población, y el incremento por la demanda de alimentos, esto ha ocasionado que exista una competencia entre humanos y animales por las trascendentales materias primas para la elaboración de piensos, provocando un rápido incremento en su costo. Este entorno obliga a los nutricionistas la búsqueda de alimentos alternativos más económicos para la alimentación de animales (1).

Por otra parte, los residuos de alimentos son principalmente de naturaleza orgánica y se caracterizan por tener elevadas cargas de nutrientes (demanda biológica de oxígeno (DBO) y una demanda química de oxígeno (DQO)) que muchas veces son descargados inadecuadamente en laderas de vías o en los sitios de generación (2).

Variedades de tratamientos se han realizado en muchos estudios recientemente, pero la mayoría enfocados a las tecnologías para la conversión en biocombustible $(3,4)$, elaboración de abonos orgánicos (5), procesos degradación enzimática convertirse en etanol por microorganismos fermentativos (6), o en tratamientos con fines energéticos renovables como la pirólisis y la digestión anaeróbica (7-9). Pero los métodos tradicionales, siguen siendo una vía empleada en los países vías de desarrollo que en muchas ocasiones no disponen de tecnologías apropiadas para los tratamientos mencionados.

Si bien la mayoría de los subproductos agroindustriales tienen un bajo contenido de nitrógeno, más fibra y una baja densidad de nutrientes, el procesamiento puede aumentar su valor nutritivo. Y como menciona la revisión de (10) son ricos en muchos compuestos bioactivos y nutracéuticos, que mediante el enriquecimiento de nutrientes y tecnologías apropiadas para su valorización pueden ser empleados como soluciones potenciales a los problemas de nutrición animal y el suministro mundial de proteínas y calorías. Además, las crecientes preocupaciones ambientales y el rápido aumento de los costos de los vertederos han llevado a la necesidad de evaluar estos productos para su uso potencial en programas de alimentación animal. La utilización de subproductos puede ser económicamente viable, pues los alimentos convencionales a menudo son costosos (11). Y los nutrientes presentes pueden ayudar a satisfacer las necesidades de cerdos en la alimentación, crecimiento, reproducción y producción.

Existen básicamente tres grupos de tecnologías para la recuperación de residuos agroindustriales: 1) la valorización biológica y química, 2) la obtención de combustibles (derivados de desechos) y 3) la valorización térmica. El primer grupo permite obtener gases, líquidos o sólidos comercializables como pectinas, enzimas, aceites esenciales, fibra dietaria (alimento para animales y humanos), hongos comestibles, flavonoides y carotenoides- a partir de residuos orgánicos. Tales productos se pueden obtener mediante procesos biológicos como el compostaje o la lombricultura, entre otros. El segundo grupo permite obtener combustibles como el biogás (utilizado para diversos fines) y el tercero 
busca la reducción del volumen de los residuos MATERIALES Y MÉTODOS y la recuperación de energía a partir de los gases, líquidos y sólidos generados, utilizando procesos como la incineración y la pirólisis

En Ecuador, existen subproductos agroindustriales viables para la alimentación animalquenoseutilizan poreldesconocimiento de sus características nutricionales (12). Sus nutrientes pueden ser materia prima para generar productos de interés, como alimento para animales (13). Algunos residuos han sido estudiados y documentados en la revisión de (14) como la utilización de cascarilla de arroz, hollejos frescos de cítricos, raíces y tubérculos frescos o cocidos. En el proceso de obtención de proteína microbiana, (15) estudia las condiciones ideales para la producción mediante fermentación en estado sólido de mezclas de desecho de manzana, con soja y alfalfa para ser empleado en la alimentación animal. Por su parte, evaluó el valor nutritivo de los ensilajes de pasto elefante (Pennisetum purpureum, Schum.) con la adición de hasta un $16 \%$ de mango (Mangifera indica, L.) obteniendo que la adición de $8.6 \%$ mejora el proceso de fermentación de los ensilajes (16).

Basado en ello, el objetivo de esta investigación fue establecer diferentes alternativas de aprovechamiento de los residuos agroindustriales en el mejoramiento de la calidad del ambiente a través del análisis proximal de residuos agroindustriales como: maní sin cáscara, maní con cáscara, cáscara de plátano, cáscara de yuca, habas sin cascara y cáscara de habas para uso en la alimentación animal. 
Tabla 1. Parámetros determinados y descripción del procedimiento.

RECURSOS

Materia Seca

Cenizas

Materia Seca

Extracto etéreo

Extractos libres de nitrógeno (ELN)

Fibra bruta

Materia orgánica:

Energía bruta
Se realizó en la estufa a $100-105^{\circ} \mathrm{C}$ durante 8 horas, verificando obtener el peso constante.

Para determinar el contenido de cenizas es necesario oxidar toda la materia orgánica de la muestra. Esta operación se efectuó en la mufla a $550^{\circ} \mathrm{C}$, durante 4 horas.

Se empleó en tres pasos: primeramente, se realizó la conversión del nitrógeno a la forma amoniacal mediante la digestión de la muestra en ácido sulfúrico ( $\mathrm{H} 2 \mathrm{SO} 4)$ en presencia de un catalizador, posteriormente se destiló el sulfato amónico ((NH4)2SO4) en una solución atrapadora, y finalmente, se cuantifico el amoníaco por valoración con una solución estándar. La proteína bruta se obtuvo de la multiplicación del nitrógeno de la muestra con el factor de conversión de 6,25.

La extracción de los materiales liposolubles se realizó con éter de petróleo a $60^{\circ} \mathrm{C}$, en el extractor Soxhlet para la evaporación del disolvente, y valoración del extracto.

Se expresó en \% y se calculó por diferencia de las otras fracciones: \% ELN $=100-(\%$ Cenizas + \% Proteína Bruta + \% Extracto etéreo + \% Fibra bruta)

Se realizaron dos hidrólisis sucesivas, una ácida, con Ácido sulfúrico 0,26 N y otra alcalina con Hidróxido Sódico 0,31 N, terminadas las hidrólisis, se lavó con agua destilada abundante y acetona, y posteriormente se secaron en la estufa a $105^{\circ} \mathrm{C}$ durante 12 horas. Se obtiene por diferencia de peso entre el residuo obtenido menos las cenizas, divido a la muestra inicial fresca.

Se obtuvo por diferencia de los inorgánicos (100 - el \% de cenizas).

Se evaluó por calorimetría, utilizando una bomba calorimétrica adiabática marca Parr, modelo 1241.

\section{Tratamiento de los datos}

Los datos de la composición química de los subproductos, se analizaron mediante el módulo estadística descriptiva, se determinó la media y desviación estándar (DE), con el empleo del programa estadístico Infostat (14).
RESULTADOS Y DISCUSIÓN

El maní sin cáscara, Tabla 2, presentó altos contenidos de MS mg (91,65\%), MO mg (96,74\%), PBmg (28,22\%), EE mg (40,49\%), EB mg $(577,68 \mathrm{kcal} 100 \mathrm{~g} \mathrm{MS}-1)$ y bajos de FB (5,85\%), ELN (22,19\%) y cenizas (3,27\%). 
Tabla 2. Composición química del maní sin cáscara.

\begin{tabular}{|c|c|c|}
\hline Variable & Media & DE \\
\hline MS, \% & 32,74 & 0,13 \\
\hline $\mathrm{MO}, \%$ & 96,85 & 0,24 \\
\hline $\mathrm{PB}, \%$ & 31,48 & 0,13 \\
\hline $\mathrm{FB}, \%$ & 0,93 & 0,23 \\
\hline $\mathrm{EE}, \%$ & 1,79 & 0,23 \\
\hline ELN, \% & 62,66 & 0,83 \\
\hline Cenizas, \% & 3,15 & 0,24 \\
\hline $\mathrm{EB}, \mathrm{kcal} 100 \mathrm{~g} \mathrm{MS}^{1}$ & 394,47 & 0,25 \\
\hline
\end{tabular}

Los subproductos de maní sin cáscara, presentaron un nivel alto de $\mathrm{PB}, \mathrm{EE}$ y EB. En la alimentación de cerdos se recomienda no incluir más del $25 \%$ de maní en la dieta por el alto contenido de EE que posee este recurso, puede tener un efecto laxante en los cerdos (17). Entre los suplementos proteicos es quizás el más palatable, lo cual, junto a su alto nivel en proteína, hacen de este subproducto un excelente suplemento para ser usado solo o en combinación con algún otro alimento de menor degradabilidad en la alimentación de animales monogástricos (18).

En la Tabla 3, se observa la composición química del maní con cáscara, este subproducto presentó altos niveles de MS (92,65\%), MO (97,47\%), PB (28\%), EE (43,19\%), EB (591,98 kcal 100 g MS-1) y bajos de FB (6,92\%), cenizas $(2,54 \%)$ y $\operatorname{ELN}(18,80 \%)$.

Tabla 3. Composición química del maní con cáscara.

\begin{tabular}{|c|c|c|}
\hline Variable & Media & DE \\
\hline MS, \% & 32,74 & 0,13 \\
\hline MO, \% & 96,85 & 0,24 \\
\hline PB, \% & 31,48 & 0,13 \\
\hline $\mathrm{FB}, \%$ & 0,93 & 0,23 \\
\hline$E E, \%$ & 1,79 & 0,23 \\
\hline ELN, \% & 62,66 & 0,83 \\
\hline Cenizas, \% & 3,15 & 0,24 \\
\hline $\mathrm{EB}, \mathrm{kcal} 100 \mathrm{~g} \mathrm{MS}^{1}$ & 394,47 & 0,25 \\
\hline
\end{tabular}


Los porcentajes de materia seca de ambos es similar a otros residuos analizados en (10), como por ejemplo la cascara de arroz que presenta valores entre $91.3-93.3 \%$, pero los valores proteicos de los subproductos de maní son superiores pues la cascara de arroz tiene como valores máximos 2,8\%. Sin embargo, la cascara de arroz presenta valores superiores de cenizas (15.6-22.6\%) y de extracto libre de nitrógeno (25-29\%).

Al igual que el maní sin cáscara, el maní con cáscara posee excelentes cualidades nutritivas para su uso en la alimentación porcina como suplemento proteico (19). Sin embargo, hay que tomar en consideración el alto nivel de grasa que posee este alimento y no hay forma de prevenir o eliminar la presencia de micotoxinas y aflatoxinas en el proceso de fabricación de alimentos balanceados, aún y cuando se incluya un "secuestrante" de micotoxinas y aflatoxinas los mismos que pueden tener efectos adversos en el desempeño de los animales $(20,21)$, por lo que el producto se debe almacenar en un lugar limpio y seco para minimizar el ingreso de estos agentes patógenos en el alimento (22).

Este aspecto de las micotoxinas es de gran interés e importancia en la producción de alimentos para animales pues son uno de los principales compuestos tóxicos que pueden encontrarse en muchos subproductos agrícolas $y$, por ende, en la alimentación animal. Generalmente metabolitos secundarios de hongos que afectan la salud humana y animal. Por su parte, las aflatoxinas son compuestos cancerígenos producidos por ciertas cepas del género Aspergillus y a menudo se encuentran como contaminantes de las dietas para rumiantes. La solución ideal para minimizar el riesgo para la salud que plantean las aflatoxinas es la prevención de la contaminación del alimento (23).

El almacenamiento y procesamiento de los productos agrícolas para producir alimento para animales debe tener un control estricto. El uso de estrategias de control de pre-cosecha, selección de variedades de resistencia, el manejo del campo, el uso de agentes biológicos y químicos, el manejo de cosecha y las aplicaciones pos-cosecha, incluida la mejora de las condiciones de secado y almacenamiento, junto con el uso de agentes naturales y químicos, y la irradiación han demostrado ser los aspectos más importantes en la prevención del crecimiento de hongos micotoxigénicos y la formación de micotoxinas (24). En el caso de las aflatoxinas un elemento a considerar puede ser el empleo de productos químicos, como el aluminosilicato, la zeolita y la bentonita han demostrado tener un buen potencial de uso en la alimentación animal para ayudar a superar la aflatoxicosis (25).

En la Tabla 4, se observa la composición química de la cáscara de plátano, este subproducto, presentó buen contenido de PB (7,93\%), MO (91,19\%), EE (5\%), ELN (72,40\%), cenizas (8,82\%), EB (378,15 kcal $100 \mathrm{~g} \mathrm{MS}-1)$ y bajos niveles de FB (5,85\%) y MS (14,06\%).

La cáscara de plátano presentó alto contenido de $M O, E L N$, cenizas, EB y bajos niveles de MS y FB. Existe elevada presencia de humedad en la cascara de plátano, cáscaras de 
mazorca de cacao estudiados en (10), presentan un $11,4 \%$ de MS, y un $6 \%$ de proteína bruta, mientras en fibra cruda el valor es superior (31.5) al de la cascara de plátano (5.85\%). En otro caso, la evaluación de las principales características de la pulpa de café fresca del estudio de (11), mostraron que los valores de Extracto etéreo, Extracto libre de Nitrógeno y Proteína cruda son inferiores 3,86\%, 60,29\% y $3,87 \%$, respectivamente; pero es superior la materia seca $87,30 \%$, las cenizas $9,12 \%$, la fibra $22,86 \%$, y en el caso de la materia orgánica son relativamente similares $(90,88 \%)$.

En la alimentación estudios de Campabadal et al., y Valdivié $(26,27)$ demostraron que es factible utilizar la harina de cáscara de plátano en un límite de inclusión de $10 \%$ en la dieta de cerdos de 10 a $20 \mathrm{~kg}$ sin afectar el comportamiento productivo de los animales.

Tabla 4. Composición química de la cáscara de plátano.

\begin{tabular}{|c|c|c|}
\hline Variable & Media & DE \\
\hline MS, \% & 32,74 & 0,13 \\
\hline MO, \% & 96,85 & 0,24 \\
\hline $\mathrm{PB}, \%$ & 31,48 & 0,13 \\
\hline $\mathrm{FB}, \%$ & 0,93 & 0,23 \\
\hline $\mathrm{EE}, \%$ & 1,79 & 0,23 \\
\hline ELN, \% & 62,66 & 0,83 \\
\hline Cenizas, \% & 3,15 & 0,24 \\
\hline EB, kcal $100 \mathrm{~g} \mathrm{MS}^{1}$ & 394,47 & 0,25 \\
\hline
\end{tabular}

En relación a la cáscara de yuca, Tabla 5 , presentó un contenido alto de MO (91,52\%), $\operatorname{ELN}(76,16 \%)$, cenizas $(8,49 \%)$, EB $(355,65 \mathrm{kcal}$ 100 g MS-1) y bajos niveles de MS (23,77 \%), FB (8,23\%), PB (5,92 \%) y EE (1,21\%).

La cáscara de yuca es una buena fuente de MO, ELN y EB. Para utilizar estos residuos en la dieta de animales monogástricos es necesario someter a métodos físicos, químicos y biológicos para mejorar las condiciones nutricionales como el aumento de proteína y digestibilidad (18), los residuos agroindustriales son una fuente importante de azúcares, almidón y carbohidratos estructurales (27). En investigaciones en cerdos (28) estableció que se puede utilizar la harina de estos subproductos con una inclusión de hasta $30 \%$ en la dieta sin afectar la ganancia de peso de los animales. 
Tabla 5. Composición química de la cáscara de yuca.

\begin{tabular}{|c|c|c|}
\hline Variable & Media & DE \\
\hline MS, \% & 32,74 & 0,13 \\
\hline $\mathrm{MO}, \%$ & 96,85 & 0,24 \\
\hline $\mathrm{PB}, \%$ & 31,48 & 0,13 \\
\hline $\mathrm{FB}, \%$ & 0,93 & 0,23 \\
\hline $\mathrm{EE}, \%$ & 1,79 & 0,23 \\
\hline ELN, \% & 62,66 & 0,83 \\
\hline Cenizas, \% & 3,15 & 0,24 \\
\hline $\mathrm{EB}, \mathrm{kcal} 100 \mathrm{~g} \mathrm{MS}^{1}$ & 394,47 & 0,25 \\
\hline
\end{tabular}

Por su parte, expone que el bagazo de caña, presenta cantidades de proteína bruta entre $2.9-6.9 \%$ y fibra cruda de $10.3-39.3 \%$, estos últimos superiores a los obtenidos en este estudio con la cascara de yuca (10).

En la Tabla 6, se observa la composición química de la cáscara de haba, este subproducto mostró alto contenido de Materia Orgánica $(97,64 \%)$, apreciable contenido de materia seca (32,74\%), Fibra Bruta (49,03\%), Extractos Libres de Nitrógeno (43,43\%), Energía Bruta $(293,28$ kcal 100 g MS-1) y bajos porcentajes de
Proteína Bruta (5,03 \%), Extracto etéreo (0,16\%) y cenizas (2,36\%).

En correspondencia al tenor de MS, MO, PB, $E E, E L N$ y EB desde el punto de vista nutricional es probable que no haya un efecto perjudicial en el desempeño de cerdos en la etapa de ceba $(29,30)$. Sin embargo, se encontró un contenido alto de fibra bruta y como menciona (29) ello influye negativamente en la especie porcina, pues mientras mayor sea su concentración menor será su aprovechamiento.

Tabla 6. Composición química de la cáscara de haba.

\begin{tabular}{|c|c|c|}
\hline Variable & Media & DE \\
\hline MS, \% & 32,74 & 0,13 \\
\hline MO, \% & 96,85 & 0,24 \\
\hline $\mathrm{PB}, \%$ & 31,48 & 0,13 \\
\hline $\mathrm{FB}, \%$ & 0,93 & 0,23 \\
\hline $\mathrm{EE}, \%$ & 1,79 & 0,23 \\
\hline ELN, \% & 62,66 & 0,83 \\
\hline Cenizas, \% & 3,15 & 0,24 \\
\hline EB, kcal 100 g MS $^{1}$ & 394,47 & 0,25 \\
\hline
\end{tabular}


Otros subproductos con valores similares de proteína bruta han sido caracterizados en otros estudios. Por ejemplo, con residuos de pulpa de cítricos obtuvo alrededor de un $6 \%$, en este caso con una gran cantidad de azúcares solubles y una digestibilidad del $85 \%$, lo que unido al contenido de proteína le permite reemplazar un tercio de la mezcla de alimento para animales sin efectos nocivos para la salud (30).

Mientras, con subproductos del mango, como los granos de semilla de mango, obtuvieron un $6 \%$ de proteína cruda y un $70 \%$ nutrientes digestibles totales y según su criterio pueden utilizarse como ingrediente en las raciones de alimentación animal (31).

Varios investigadores manifiestan que no es una limitante la utilización de subproductos fibrosos, si se utilizan procesos biotecnológicos para mejorar el valor nutritivo de estos alimentos cuando se emplean como materia prima para la obtención de alimentos para animales (32), por la acción de los microorganismos presentes en este proceso, bacterias y hongos (33). Este efecto se debe a la capacidad de producción de enzimas por parte de los microorganismos y entre las más importantes se encuentran; $\alpha$-amilasa, arabinosa, celulasa, dextranasa, y $\beta$-glucanasa (34).

La composición química de las habas sin cáscara se observa en la Tabla 7, este subproducto presentó buen contenido de MS (32\%), MO (96,85\%), PB (31,48\%), ELN (62,66\%), EB $(394,47$ kcal 100 g MS-1) y bajo tenor de FB (0,93\%), EE (1,79\%) y cenizas $(3,15 \%)$.

Tabla 7. Composición química del haba sin cáscara.

\begin{tabular}{|c|c|c|}
\hline Variable & Media & DE \\
\hline MS, \% & 32,74 & 0,13 \\
\hline MO, \% & 96,85 & 0,24 \\
\hline $\mathrm{PB}, \%$ & 31,48 & 0,13 \\
\hline $\mathrm{FB}, \%$ & 0,93 & 0,23 \\
\hline$E E, \%$ & 1,79 & 0,23 \\
\hline ELN, \% & 62,66 & 0,83 \\
\hline Cenizas, \% & 3,15 & 0,24 \\
\hline $\mathrm{EB}, \mathrm{kcal} 100 \mathrm{~g} \mathrm{MS}^{1}$ & 394,47 & 0,25 \\
\hline
\end{tabular}

El haba sin cáscara es una buena fuente de MO, PB y ELN. Entre los factores que hay que tener en consideración para el aprovechamiento de este subproducto es el contenido de metabolitos secundarios que poseen estos alimentos (35). En animales monogástricos se debe tomar en consideración el límite de inclusión de estas materias primas para no afectar el consumo y con ello el normal desempeño de los animales (36). 


\section{CONCLUSIONES}

Los residuos de maní (sin cáscara y con cáscara) mostraron los mayores contenidos de materia seca, proteína bruta, extracto etéreo y energía bruta. Por su parte, el mejor resultado de los extractos libres de nitrógeno corresponde a las habas sin cáscara con un $62,66 \%$. El mayor contenido de fibra bruta se encontró en la cáscara de haba con un 49,03 $\%$, mientras los mayores resultados en cuanto a cenizas fueron los residuos de la cáscara de plátano y yuca $(8,82$ y $8,49 \%)$ respectivamente. Los subproductos agroindustriales de maní sin cáscara, maní con cáscara, cáscara de plátano, cáscara de yuca, cáscara de habas y habas sin cáscara presentaron buen contenido de materia seca, materia orgánica, proteína bruta, extractos libres de nitrógeno, cenizas, extracto etéreo y energía bruta, todos aptos para uso en la alimentación porcina. Se recomienda evaluar estos subproductos en estudios con cerdos para cuantificar los aspectos nutritivos, y valorar la factibilidad de su uso como alimento animal

\section{REFERENCIAS BIBLIOGRÁFICAS}

1. Abdulrashid M. y Nnabuenyi, L. Taro Cocoyam (Colocacia esculenta) Meal as Feed Ingredient in Poultry. Pakistan Journal of Nutrition. 2009, 8(5): 668-673

2. Ravindran R., \& Jaiswal, A. K. Exploitation of food industry waste for high-value products. Trends in Biotechnology. 2009, 34(1), 58-69. DOI: https://doi.org/10.1016/j. tibtech.2015.10.008

3. Parmar I. and Rupasinghe, H.V. Bioconversion of apple pomace into ethanol and acetic acid: enzymatic hydrolysis and fermentation. Bioresour. Technol. 2013, 130,
613-620. DOI: https://doi.org/10.1016/j. biortech.2012.12.084

4. Liguori R. et al. Second generation ethanol production from brewers' spent grain. Energies. 2013, 8, 2575-2586

5. Caiza D, Chimbo A, Pereira L, BS, Pisco, WE, y Diéguez-Santana K. Propuesta de producción más limpia en el proceso de elaboración de abonos orgánicos con desechos del camal, realizado en el relleno sanitario del cantón Baños de Agua Santa, provincia de Tungurahua. Revista Observatorio de la Economía Latinoamericana. 2018. En línea: https://www.eumed.net/rev/oel/2018/06/ elaboracion-abonos-organicos.html

6. Das SP, Ravindran R, Ahmed S. et al. Bioethanol production involving recombinant C. thermocellum hydrolytic hemicellulase and fermentative microbes. Appl. Biochem. Biotechnol. 2012;167, 1475-1488. DOI: https:// doi.org/10.1007/s12010-012-9618-7

7. Azadi $P$ et al. Liquid fuels, hydrogen and chemicals from lignin: a critical review. Renew. Sust. Energ. Rev. 2013; 21, 506-523. DOI: https://doi.org/10.1016/j.rser.2012.12.022

8. Arteaga-Pérez LE, Segura C, \& Santana KD. Procesos de torrefacción para valorización de residuos lignocelulósicos. Análisis de posibles tecnologías de aplicación en Sudamérica. Afinidad, 2016; 73(573).

9. González EC, Pérez-Martínez A, Salabarría JL, \& Diéguez-Santana K. Relationship between the fed substrates and the physical chemical parameters of an anaerobic biodigester in Ecuadorian Amazon Region. In MOL2NET 2017, International Conference on Multidisciplinary Sciences, 2017; 3rd edition (p. 5039). MDPI AG

10. Ajila CM, Brar SK, Verma M, Tyagi RD, Godbout S, \& Valéro JR. Bio-processing of agro-byproducts to animal feed. Critical reviews in biotechnology, 2012; 32(4), 382400. DOI: 10.3109/07388551.2012.659172

11. Noriega A, Silva R. y García M. Utilización de la pulpa de café en la alimentación animal. 
Revista Zootecnia tropical. 2008; 26(4): 411419

12. Caicedo W. Tubérculos de papa china (Colocasia esculenta (L,) Schott) como una fuenteenergética tropical paraalimentarcerdos. Una reseña corta sobre las características de la composición química y de los factores antinutricionales. Revista Computadorizada de Producción Porcina. 2013; 20: 278-282.

13. Domínguez $\mathrm{PL}$, Chao $\mathrm{R}$, Víctores $\mathrm{N}, \mathrm{y}$ Herrera R. Utilización digestiva y balance de $\mathrm{N}$ de ensilado de cuerpos de cerdos para el ganado porcino. Revista Computadorizada de Producción Animal. 2012; 19(2): 128-130.

14. Martin P. El uso de residuales agroindustriales en la alimentación animal en Cuba: pasado, presente y futuro. Revista Avances en Investigación Agropecuaria. 2009; 13(3): 3-10.

15. Díaz D, Rodríguez $C$, Mancillas $P$, Angulo $C$, Salvador F, Arzola C, Jiménez J, Mena S y Elías A. Producción de proteína microbiana a partir de manzana de desecho con fermentación en estado sólido a $32^{\circ} \mathrm{C}$. Revista electrónica de Veterinaria. 2010; 11(10): 1-9

16. Rego $M$, Neiva J, Rego A, Cándido $M$, Carneiro $M$, y Lobo R. Chemical and bromatological characteristics of elephant grass silages containing a mango byproduct. 2010; Rev. Brasileira de Zootecnia 39:81. DOI: http://dx.doi.org/10.1590/S151635982010000100011

17. Rostagno HS, Teixeira LF, Donzele LJ, Gomes PC, Oliverira Rita, Lopes DC, Ferreira AS, Toledo SL, y Euclides RF. Tablas Brasileñas para aves y cerdos. Composición de Alimentos y Requerimientos Nutricionales. 2011; 3era Edición. Universidad Federal de Viçosa - Departamento de Zootecnia, Brasil, Pp 167.

18. Caicedo WO, Rodríguez R, Lezcano $P$, y Ly J. Estudios de composición química de ensilados de papa china (Colocasia esculenta L. Schott) destinados a la alimentación porcina. In: XXIII Reunión de la Asociación
Latinoamericana de Producción Animal. La Habana, versión electrónica disponible en disco compacto. 2013; ISBN 978950717149 2

19. Pozza P, Gomes P, Donzele J, Rostagno H, Pozza M, y Lopes D. Digestibilidades Ileal Aparente e Verdadeira dos Aminoácidos de Farinhas de Vísceras para Suínos. R. Bras. Zootec., 200534(6): 2327-2334

20. Etienne M, y Dourmad JY. Effects of zearalenone or glucosinolates in the diet on reproduction in sows. A review. Livestock Production Science. 1994; 40:99-113

21. Schwarzer K. Reducing zearalenone impact on semen quality. Pig Progress. 2002; 18(5):33-35

22. Jouany JP. Methods for preventing, decontaminating and minimizing the toxicity of mycotoxins in feeds'. Anim. Feed Sci. Technol. 2007; 137: 342-362. DOI: https://doi. org/10.1016/j.anifeedsci.2007.06.009

23. Saima M, ZU, Khan TN, Pasha TN, Jabbar MA. Application of biotechnology to enhance nutritive value of low quality agricultural residues in buffalo ration: AReview. Pakistan J Zool Suppl Ser, 2009; 9: 441-446

24. Milicevic DR, Skrinjar M, Baltic T. Real and perceived risks for mycotoxin contamination in foods and feeds: Challenges for food safety control. 2010; Toxins (Basel) 2: 572-592.

25. Kabak B, Dobson AD, Var I. A. Strategies to prevent mycotoxin contamination of food and animal feed: review. 2006; Crit Rev Food Sci Nutr 46: 593-619

26. Campabadal C, Solís J, y Molina JR. Evaluación de diferentes formas de suplir el banano en la alimentación de cerdos, durante la etapa de crecimiento y engorde. Agronomía Costarricense. 1988; 12: 2

27. Gómez J, Yepes S, y Barahona R. Caracterización nutricional del residuo del cultivo de la seta Agaricus bisporus como alimento potencial para bovinos. Revista CES Medicina Veterinaria y Zootecnia. 2013; 8(1): 34-56 
28. Buitrago JA. La yuca en la alimentación animal. Centro Internacional de Agricultura Topical. Colombia. 1990; Pp. 165

29. Almaguel R, Lezcano P, Delgado E, y Ly J. Miel rica o maíz como fuente de energía para cerdos en crecimiento. Rasgos de comportamiento y digestibilidad rectal. In: XXIII Reunión de La Asociación Latinoamericana de Producción Animal (ALPA). La Habana. 2013; ISBN 9789597171492

30. Almaguel RE, Cruz E, Castro M, y Ly J. Balance de $\mathrm{N}$ y Energía en Cerdos Alimentados con Dietas de Mieles de Caña de Azúcar y Cachaza Biotransformada (GARANVER). Revista Computadorizada de Producción Porcina. 2008; 15 (4):338-341

31. Ly J, Reyes JL, Macías M, Martínez V, Domínguez PL, y Ruiz R. Ileal and total tract digestibility of leucaena meal (Leucaena leucocephala Lam. De Wit) in growing pigs. Animal Feed Science and Technology. 1998; 70:265-273. DOI: https://doi.org/10.1016/ S0377-8401(96)01106-6

32. Guzmán, O., Lemus, C., Martínez, S., Bonilla, J., Plasencia, A. y Ly, J. Chemical characteristics of silages of mango (Mangifera indica L.) byproducts for animal feeding.
Cuban Journal of Agricultural Science, 2012; 46(4). Retrieved from http://cjascience.com/ index.php/CJAS/article/view/148

33. Paredes D, Álvarez M, y Silva M. Obtención de Enzimas Celulasas por Fermentación Sólida de Hongos para ser Utilizadas en el Proceso de Obtención de Bioalcohol de Residuos del Cultivo de Banano. Revista Tecnológica ESPOL-RTE. 2010; 23(1): 81-88

34. Giang HH, Viet TQ, Ogle B. y Lindberg JE. Effects of Supplementation of Probiotics on the Performance, Nutrient Digestibility and Faecal Microflora in Growing-finishing Pigs. AsianAust. J. Anim. Sci. 2011; 24(5):655-661

35. Buntha P, Borin K, Preston TR, y Ogle B. Digestibility and nitrogen balance studies in pigs fed diets with ensiled taro (Colocasia esculenta) leaves as replacement for fish meal. Livestock Research for Rural Development 20, 2008; Available: <http://library.wur.nl/WebQuery/ clc/1740797>, [Accessed: February 26, 2017]. 36. Lezcano P, Berto D, Bicudo S, Curcelli F, Figueiredo $P$, y Valdivie M. Yuca

ensilada como fuente de energía para cerdos en crecimiento. Avances en Investigación

Agropecuaria. 2014; 18(3):41-47 